\title{
Profit rate performance optimization for a generalized irreversible combined refrigeration cycle
}

\author{
KANG MA, LINGEN CHEN*, and FENGRUI SUN \\ Postgraduate School, Naval University of Engineering, Wuhan 430033, P. R. China \\ e-mail:1gchenna@yahoo.com and lingenchen@hotmail.com
}

MS received 23 November 2008; revised 13 April 2009

\begin{abstract}
Finite-time exergoeconomic performance of a Newtonian heat transfer law system generalized irreversible combined refrigeration cycle model with finite-rate heat transfer, heat leakage and internal irreversibility is presented in this paper. The operation of the generalized irreversible combined refrigeration cycle is viewed as a production process with exergy as its output. The performance optimization of the cycle is performed by taking profit as the objective. The optimal profit rate, optimal COP (coefficient of performance), as well as the relation between the optimal profit rate and COP of the cycle are derived. The focus of this paper is to obtain the compromise optimization between economics (profit rate) and the energy utilization factor (COP) for the cycle, by searching the optimum $\mathrm{COP}$ at maximum profit rate, which is termed as the finite time exergoeconomic performance bound. Moreover, the effects of various factors, including heat leakage, internal irreversibility and the price ratio, on the profit rate performance of the cycle are analysed by detailed numerical examples.
\end{abstract}

Keywords. Finite-time thermodynamics; generalized irreversible combined refrigeration cycle; exergoeconomic performance; generalized thermodynamic optimization.

\section{Introduction}

Since finite time thermodynamics and entropy generation minimization has been advanced (Andresen 1983; Bejan 1996; Berry et al 1999; Chen et al 1999a; Chen \& Sun 2004; Chen 2005; Durmayaz et al 2004; Sieniutycz \& Salamon 1990), the research into identifying the performance limits of thermodynamic processes and optimization of thermodynamic cycles has been made a tremendous progress by scientists and engineers. The objective functions in finite time thermodynamics are often pure thermodynamic parameters including power, efficiency, entropy production, effectiveness, cooling load, heating load, coefficient of performance (COP), loss of exergy, etc. Berry \& Salamon (1978) carried out the economic optimization of the heat engine with the maximum profit rate as the objective function.

*For correspondence 
Salamon \& Nitzan (1981) viewed the operation of the endoreversible heat engine as a production process with work as its output.

A relatively new method that combines exergy with conventional concepts from long-run engineering economic optimization to evaluate and optimize the design and performance of energy systems is exergoeconomic (or thermoeconomic) analysis (Tsatsaronts 1993; El-Sayed 2003). Salamon \& Nitzan (1981) combined the endoreversible model with exergoeconomic analysis. It was termed as finite time exergoeconomic analysis to distinguish it from the endoreversible analysis with pure thermodynamic objectives and the exergoeconomic analysis with long-run economic optimization (Chen et al 1991, 1996, 2001, 2004a; Wu et al 1996, 1998; Wu et al 2000; Zheng et al 2006). Similarly, the performance bound at maximum profit rate was termed as finite time exergoeconomic performance bound to distinguish it from the finite time thermodynamic performance bound at maximum thermodynamic output. A similar idea was provided by Ibrahim et al (1992), De Vos $(1995,1997)$ and Bejan (1993). De Vos $(1995,1997)$ used the basic idea of finite time thermodynamics into the thermoeconomics for heat engine in which the heat transfer between the working fluid and the heat reservoirs obeys Newtonian heat transfer law, derived the relation between the optimal efficiency and economic returns. Chen et al (2005) investigated the endoreversible thermoeconomic performance of heat engine with the linear phenomenological heat transfer law based on the work of De Vos (1995). Scholars provided a new thermoeconomic optimization criterion, thermodynamic output rates (power, cooling load or heating load for heat engine, refrigerator or heat pump) per unit total cost, investigated the performances of endoreversible heat engine (Sahin \& Kodal 2001), refrigerator and heat pump (Sahin \& Kodal 1999), combined cycle refrigerator (Sahin \& Kodal 2002), combined cycle heat pump (Kodal et al 2000b), as well as irreversible heat engine (Kodal \& Sahin 2003), refrigerator and heat pump (Kodal et al 2000a), combined cycle refrigerator (Sahin et al 2001), combined cycle heat pump (Kodal et al 2002), and threeheat- reservoir absorption refrigerator and heat pump (Kodal et al 2003). This method was also applied to the optimization of an endoreversible four-heat-reservoir absorption-refrigerator by Qin et al (2005).

Some authors have analysed the influences of heat resistance (Chen \& Yan 1988; Chen et al 1995), heat resistance and internal irreversibility (Goktun 1996), and heat resistance and heat leakage (Chen et al 1997) on the performance of combined refrigeration cycles. Chen et al (1999b) established a generalized irreversible combined refrigeration cycle model with heat resistance, heat leakage and internal irreversibility, and derived the analytical formulae of optimal cooling load and COP of the refrigeration cycle model. A further step made in this paper is to study the finite time exergoeconomic performance of the generalized irreversible combined refrigeration cycle. The analytical formulae about optimal profit rate, optimal COP and relation between the optimal profit rate and $\mathrm{COP}$ of the cycle with Newtonian heat transfer law are derived. The aim of this paper is to obtain the compromise optimization between economics (profit rate) and the energy utilization factor (COP) for the cycle, by searching the optimum COP at maximum profit rate which is termed as the finite time exergoeconomic performance bound. Moreover, analysis and optimization of the model are carried out in order to investigate the effects of cycle parameters on the performance of the cycle using numerical examples.

\section{Model of the combined refrigeration cycle (Chen et al 1999b)}

A combined refrigeration cycle formed by two steady irreversible Carnot refrigeration cycles and its surrounding heat reservoirs are shown in figure 1 . The combined cycle operates between 


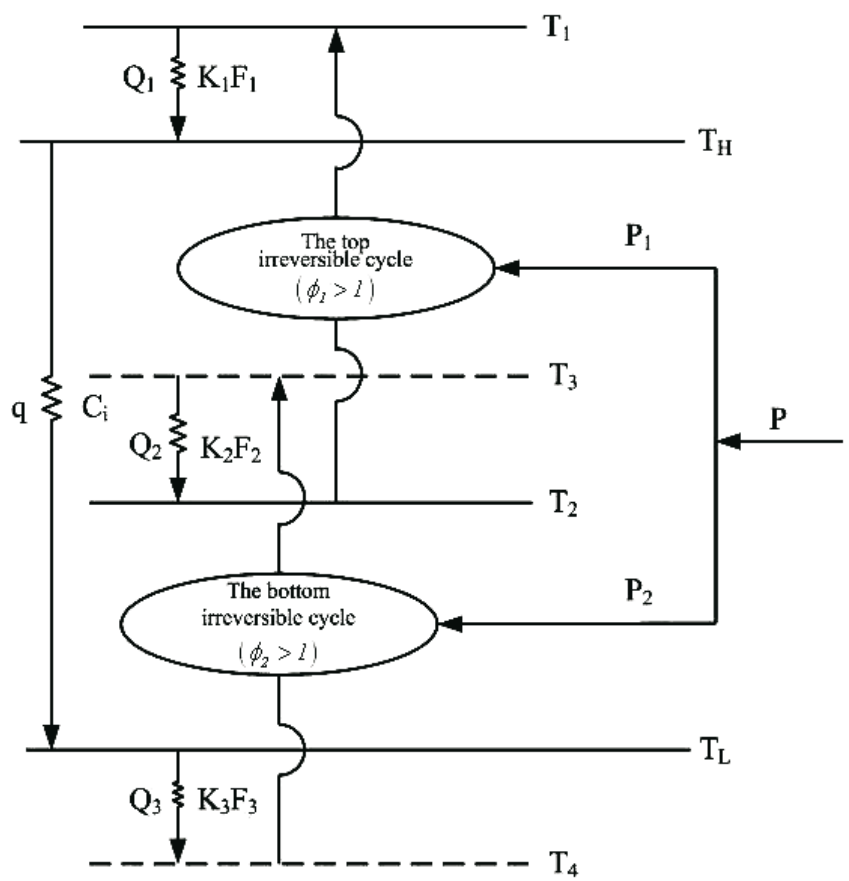

Figure 1. Generalized irreversible combined Carnot refrigeration cycle model.

two reservoirs at temperatures $T_{H}$ and $T_{L}\left(T_{H}>T_{L}\right)$. The temperatures of the working fluid in the top irreversible cycle are $T_{1}$ and $T_{2}$, when heat leaves and enters the working fluid. In the bottom irreversible cycle, they are $T_{3}$ and $T_{4}$. These temperatures are related to one another in the following order:

$$
T_{1}>T_{H}>T_{3}>T_{2}>T_{L}>T_{4} .
$$

Heat exchange between the two irreversible cycles is carried out directly through the same heat transfer surface area so that the quantity of heat absorbed by the working fluid in the top cycle is equal to that rejected by the working fluid in the bottom cycle. The irreversibilities are the constant rate of bypass heat leak $q$, defined by Bejan $(1988,1989)$, which flows from the heat sink at temperature $T_{H}$ to the heat source at temperature $T_{L}$, the internal dissipation of the working fluids in the top and bottom cycles, and the three heat transfer losses caused by the three finite temperature differences $\left(T_{1}-T_{H}\right),\left(T_{3}-T_{2}\right)$ and $\left(T_{L}-T_{4}\right)$ required by the heat rejection and absorption rates.

The first law of thermodynamics requires

$$
Q_{H}=Q_{1}-q, R=Q_{L}=Q_{3}-q,
$$

where $Q_{1}$ and $Q_{3}$ are the rates of heat flow from the top cycle to the heat sink in the hightemperature side heat exchanger and from the heat source to the bottom cycle in the lowtemperature side heat exchanger of the combined cycle, respectively, $Q_{H}$ and $Q_{L}$ are the heat transfer rate to the heat sink and the heat removed rate from the heat source (i.e. cooling load, $R$, for convenience), respectively.

The internal dissipation including miscellaneous factors such as friction, turbulence and non-equilibrium activities inside the two cycles are characterized by two constant coefficients 
(Chen et al 1999b):

$$
\phi_{1}=Q_{1} / Q_{1}^{\prime} \geq 1, \phi_{2}=Q_{2} / Q_{2}^{\prime} \geq 1,
$$

where $Q_{2}$ is the rate of heat exchange between the top and bottom irreversible cycles in the intermediate heat exchanger, $Q^{\prime}{ }_{2}$ is the rate of heat absorbed from the bottom endoreversible cycle to the top irreversible cycle in the intermediate heat exchanger, and $Q^{\prime}{ }_{1}$ is the rate of heat rejected from the top endoreversible cycle to the heat sink in the high-temperature side heat exchanger. Because the power input required by the irreversible refrigeration cycle is larger than that of the endoreversible one with the same cooling load, the rate of heat rejected from the warm working fluid to the heat sink for the irreversible cycle is larger than that for the endoreversible one. Thus, $Q_{1}>Q_{1}^{\prime}$ and $Q_{2}>Q_{2}^{\prime}$ hold. For the top endoreversible refrigeration cycle, the second law of thermodynamics requires that (no matter whether the bottom refrigeration cycle is endoreversible)

$$
Q_{2} / Q_{1}^{\prime}=T_{2} / T_{1} \text {. }
$$

For the bottom endoreversible refrigeration cycle, the second law of thermodynamics requires that (no matter whether the top refrigeration cycle is endoreversible)

$$
Q_{3} / Q_{2}^{\prime}=T_{4} / T_{3}
$$

The power input of the irreversible combined refrigeration cycle is:

$$
P=P_{1}+P_{2}=Q_{H}-Q_{L}=Q_{1}-Q_{3},
$$

where $P_{1}, P_{2}$ and $P$ are the power inputs to the top and bottom cycles and the total power input to the irreversible combined refrigeration cycle.

The COP of the irreversible combined refrigeration cycle is:

$$
\varepsilon=Q_{L} / P=\left(1-q / Q_{3}\right) /\left(Q_{1} / Q_{3}-1\right)
$$

Assuming that the rate of heat flow in the heat exchangers follows the Newtonian law, one has

$$
\begin{aligned}
& Q_{1}=\left(T_{1}-T_{H}\right) K_{1} F_{1}=\left(T_{1}-T_{H}\right) U_{1} \\
& Q_{2}=\left(T_{3}-T_{2}\right) K_{2} F_{2}=\left(T_{3}-T_{2}\right) U_{2} \\
& Q_{3}=\left(T_{L}-T_{4}\right) K_{3} F_{3}=\left(T_{L}-T_{4}\right) U_{3},
\end{aligned}
$$

where $K_{1}$ is the overall heat transfer coefficient and $F_{1}$ is the heat transfer surface area of the high-temperature (hot) side heat exchanger between the top cycle and the heat sink, $K_{2}$ is the overall heat transfer coefficient and $F_{2}$ is the heat transfer surface area of the intermediate heat exchanger between the top and bottom cycles, and $K_{3}$ is the overall heat transfer coefficient and $F_{3}$ is the heat transfer surface area of the low-temperature (cold) side heat exchanger between the bottom cycle and the heat source. $U$ is the heat exchanger inventory: $U_{1}=K_{1} F_{1}$, $U_{2}=K_{2} F_{2}$, and $U_{3}=K_{3} F_{3}$.

The total heat transfer surface area $\left(F_{T}\right)$ of the combined cycle is assumed to be a constant

$$
F_{1}+F_{2}+F_{3}=F_{T} .
$$




\section{Analysis and optimization}

Combining Eqs. (2)-(10), one has

$$
Q_{1}=\frac{\phi_{T} T_{H}(R+q)}{T_{L}-(R+q)\left[\left(K_{1} F_{1} / \phi_{T}\right)^{-1}+\left(K_{2} F_{2} / \phi_{2}\right)^{-1}+\left(K_{3} F_{3}\right)^{-1}\right]},
$$

where $\phi_{T}$ is the total degree of internal dissipation in the combined system,

$$
\phi_{T}=\phi_{1} \phi_{2}
$$

Substituting Eqs. (8), (10) and (12) into Eq. (7) yields (Chen et al 1999b)

$\varepsilon=\frac{R}{R+q}\left\{\frac{\phi_{T} T_{H}}{T_{L}-(R+q)\left[\left(K_{1} F_{1} / \phi_{T}\right)^{-1}+\left(K_{2} F_{2} / \phi_{2}\right)^{-1}+\left(K_{3} F_{3}\right)^{-1}\right]}-1\right\}^{-1}$.

Eq. (14) provides a general relation between the COP and the cooling load of the irreversible combined refrigeration cycle.

Assuming the environmental temperature is $T_{0}$, the rate of exergy input of the refrigeration cycle is:

$$
A_{\mathrm{rev}}=Q_{L}\left(T_{0} / T_{L}-1\right)-Q_{H}\left(T_{0} / T_{H}-1\right)=Q_{L} \eta_{2}-Q_{H} \eta_{1},
$$

where $\eta_{i}$ is the Carnot coefficient of the heat reservoir $i$, and defined as $\eta_{1}=T_{0} / T_{H}-1$ and $\eta_{2}=T_{0} / T_{L}-1$.

Assuming that the prices of exergy output rate and the power input be $\psi_{1}$ and $\psi_{2}$, the profit rate of the refrigeration cycle is:

$$
\pi=\psi_{1} A_{\text {rev }}-\psi_{2} P .
$$

Substituting Eqs. (2), (6), (9), (14) and (15) into Eq. (16) yields

$$
\pi=\kappa_{2} R+\kappa_{1} q-\frac{\kappa_{1} \phi_{T} T_{H}(R+q)}{T_{L}-(R+q)\left[\left(K_{1} F_{1} / \phi_{T}\right)^{-1}+\left(K_{2} F_{2} / \phi_{2}\right)^{-1}+\left(K_{3} F_{3}\right)^{-1}\right]},
$$

where $\kappa_{1}=\left(\psi_{1} \eta_{1}+\psi_{2}\right)$ and $\kappa_{2}=\left(\psi_{1} \eta_{2}+\psi_{2}\right)$.

Obviously, both the COP $(\varepsilon)$ and profit rate $(\pi)$ of the combined refrigeration cycle are functions of $F_{1}, F_{2}$ and $F_{3}$ for fixed $T_{H}, T_{L}, \psi_{1}, \psi_{2}, \eta_{1}, \eta_{2}, \phi_{1}, \phi_{2}, K_{1}, K_{2}, K_{3}, q$ and $R$.

With the constraint of Eq. (11), one may obtain, when

$$
\begin{aligned}
& \left(\frac{F_{1}}{F_{T}}\right)_{\mathrm{opt}}=\left(\frac{\phi_{T}}{K_{1}}\right)^{\frac{1}{2}}\left[\left(\frac{1}{K_{3}}\right)^{\frac{1}{2}}+\left(\frac{\phi_{2}}{K_{2}}\right)^{\frac{1}{2}}+\left(\frac{\phi_{T}}{K_{1}}\right)^{\frac{1}{2}}\right]^{-1} \\
& \left(\frac{F_{2}}{F_{T}}\right)_{\mathrm{opt}}=\left(\frac{\phi_{2}}{K_{2}}\right)^{\frac{1}{2}}\left[\left(\frac{1}{K_{3}}\right)^{\frac{1}{2}}+\left(\frac{\phi_{2}}{K_{2}}\right)^{\frac{1}{2}}+\left(\frac{\phi_{T}}{K_{1}}\right)^{\frac{1}{2}}\right]^{-1} \\
& \left(\frac{F_{3}}{F_{T}}\right)_{\mathrm{opt}}=\left(\frac{1}{K_{3}}\right)^{\frac{1}{2}}\left[\left(\frac{1}{K_{3}}\right)^{\frac{1}{2}}+\left(\frac{\phi_{2}}{K_{2}}\right)^{\frac{1}{2}}+\left(\frac{\phi_{T}}{K_{1}}\right)^{\frac{1}{2}}\right]^{-1}
\end{aligned}
$$


the optimal COP and profit rate of the combined refrigeration cycle are in the following forms, respectively

$$
\begin{aligned}
\varepsilon= & \frac{\left(T_{L}-q B^{*}\right) R-B^{*} R^{2}}{B^{*} R^{2}+\left(\phi_{T} T_{H}-T_{L}+2 q B^{*}\right) R+\left(\phi_{T} T_{H}-T_{L}+q B^{*}\right) q} \\
\pi= & \psi_{1} q\left(\eta_{1}-\eta_{2}\right) \\
& +\frac{-\kappa_{2} B^{*} R^{2}+\left(\kappa_{2} T_{L}-2 \kappa_{2} B^{*} q-\kappa_{1} \phi_{T} T_{H}\right) R+\left(\kappa_{2} T_{L}-\kappa_{2} B^{*} q-\kappa_{1} \phi_{T} T_{H}\right) q}{T_{L}-(R+q) B^{*}}
\end{aligned}
$$

where $B^{*}=\left[\left(1 / K_{3}\right)^{1 / 2}+\left(\phi_{2} / K_{2}\right)^{1 / 2}+\left(\phi_{T} / K_{1}\right)^{1 / 2}\right]^{2} / F_{T}$.

Combining Eqs. (21) with (22), by eliminating the cooling load $R$, one can obtain the relation between the optimal COP and profit rate of the combined cycle in the following forms

$$
\begin{aligned}
& \left\{\left[\varepsilon\left(\kappa_{2}-\kappa_{1}\right)-\kappa_{1}\right] T_{L}-\left[\pi \varepsilon+q \varepsilon\left(\kappa_{2}-\kappa_{1}\right)-q \kappa_{1}\right] B^{*}\right\}\left[\pi \varepsilon+q \varepsilon\left(\kappa_{2}-\kappa_{1}\right)-q \kappa_{1}+\pi\right] \\
& -\phi_{T} T_{H}\left[\pi \varepsilon+q \varepsilon\left(\kappa_{2}-\kappa_{1}\right)-q \kappa_{1}\right]\left[\varepsilon\left(\kappa_{2}-\kappa_{1}\right)-\kappa_{1}\right]=0 .
\end{aligned}
$$

From Eq. (22), one may obtain that $\pi=\psi_{1} q\left(\eta_{1}-\eta_{2}\right)$ when $R=\left[\kappa_{1} \phi_{T} T_{H}-\right.$ $\left.\kappa_{2}\left(T_{L}-q B^{*}\right)\right] / \kappa_{2} B^{*}$ and $R=-q$. It can be proved using Rolle's Theorem that there exists a maximum profit rate point. To find the maximum profit rate, taking the derivation of $\pi$ with respect to $R$ and setting it equal to zero $(d \pi / d R=0)$, one can obtain the maximum profit rate $\left(\pi_{\max }\right)$ and the corresponding cooling load $R_{\pi}$ as follows.

$$
\begin{aligned}
\pi_{\max } & =\psi_{1} q\left(\eta_{1}-\eta_{2}\right)+\frac{\left[T_{L}-\left(\kappa_{1} \phi_{T} T_{H} T_{L} / \kappa_{2}\right)^{\frac{1}{2}}\right]\left[\left(\kappa_{1} \kappa_{2} \phi_{T} T_{H} T_{L}\right)^{\frac{1}{2}}-\kappa_{1} \phi_{T} T_{H}\right]}{B^{*}\left(\kappa_{1} \phi_{T} T_{H} T_{L} / \kappa_{2}\right)^{\frac{1}{2}}} \\
R_{\pi} & =\frac{T_{L}-\left(\kappa_{1} \phi_{T} T_{H} T_{L} / \kappa_{2}\right)^{\frac{1}{2}}}{B^{*}}-q .
\end{aligned}
$$

Substituting Eq. (25) into Eq. (21) gives $\varepsilon_{\pi}$, which is the finite-time exergoeconomic bound of the generalized irreversible combined refrigeration cycle:

$$
\varepsilon_{\pi}=\frac{\left(\kappa_{1} \phi_{T} T_{H} T_{L} / \kappa_{2}\right)^{\frac{1}{2}}\left[T_{L}-q B^{*}-\left(\kappa_{1} \phi_{T} T_{H} T_{L} / \kappa_{2}\right)^{\frac{1}{2}}\right]}{\left[T_{L}-\left(\kappa_{1} \phi_{T} T_{H} T_{L} / \kappa_{2}\right)^{\frac{1}{2}}\right]\left[\phi_{T} T_{H}-\left(\kappa_{1} \phi_{T} T_{H} T_{L} / \kappa_{2}\right)^{\frac{1}{2}}\right]} .
$$

\section{Discussions}

If $\phi_{1}=\phi_{2}=1$, Eqs. (21), (22), (25), (24) and (26) become

$$
\begin{aligned}
\varepsilon & =\frac{\left(T_{L}-q B_{1}^{*}\right) R-B_{1}^{*} R^{2}}{B_{1}^{*} R^{2}+\left(T_{H}-T_{L}+2 q B_{1}^{*}\right) R+\left(T_{H}-T_{L}+q B_{1}^{*}\right) q} \\
\pi & =\psi_{1} q\left(\eta_{1}-\eta_{2}\right)+\frac{(R+q)\left\{\kappa_{2}\left[T_{L}-(R+q) B_{1}^{*}\right]-\kappa_{1} T_{H}\right\}}{T_{L}-(R+q) B_{1}^{*}} \\
R_{\pi} & =\frac{T_{L}-\left(\kappa_{1} T_{H} T_{L} / \kappa_{2}\right)^{\frac{1}{2}}}{B_{1}^{*}}-q
\end{aligned}
$$




$$
\begin{aligned}
\pi_{\max } & =\psi_{1} q\left(\eta_{1}-\eta_{2}\right)+\frac{\left[T_{L}-\left(\kappa_{1} T_{H} T_{L} / \kappa_{2}\right)^{\frac{1}{2}}\right]\left[\left(\kappa_{1} \kappa_{2} T_{H} T_{L}\right)^{\frac{1}{2}}-\kappa_{1} T_{H}\right]}{B_{1}^{*}\left(\kappa_{1} T_{H} T_{L} / \kappa_{2}\right)^{\frac{1}{2}}} \\
\varepsilon_{\pi} & =\frac{\left(\kappa_{1} T_{H} T_{L} / \kappa_{2}\right)^{\frac{1}{2}}\left[T_{L}-q B_{1}^{*}-\left(\kappa_{1} T_{H} T_{L} / \kappa_{2}\right)^{\frac{1}{2}}\right]}{\left[T_{L}-\left(\kappa_{1} T_{H} T_{L} / \kappa_{2}\right)^{\frac{1}{2}}\right]\left[T_{H}-\left(\kappa_{1} T_{H} T_{L} / \kappa_{2}\right)^{\frac{1}{2}}\right]}
\end{aligned}
$$

where $B_{1}^{*}=\left[\left(1 / K_{1}\right)^{1 / 2}+\left(1 / K_{2}\right)^{1 / 2}+\left(1 / K_{3}\right)^{1 / 2}\right]^{2} / F_{T}$.

Eq. (31) is the finite-time exergoeconomic performance bound of the irreversible combined refrigeration cycle with losses of heat resistance and heat leakage.

If $\phi_{T}>1$ and $q=0$, Eqs. (25), (24) and (26) becomes

$$
\begin{aligned}
R_{\pi} & =\frac{T_{L}-\left(\kappa_{1} \phi_{T} T_{H} T_{L} / \kappa_{2}\right)^{\frac{1}{2}}}{B^{*}} \\
\pi_{\max } & =\frac{\left[T_{L}-\left(\kappa_{1} \phi_{T} T_{H} T_{L} / \kappa_{2}\right)^{\frac{1}{2}}\right]\left[\left(\kappa_{1} \kappa_{2} \phi_{T} T_{H} T_{L}\right)^{\frac{1}{2}}-\kappa_{1} \phi_{T} T_{H}\right]}{B^{*}\left(\kappa_{1} \phi_{T} T_{H} T_{L} / \kappa_{2}\right)^{\frac{1}{2}}} \\
\varepsilon_{\pi} & =\frac{\left(\kappa_{1} \phi_{T} T_{H} T_{L} / \kappa_{2}\right)^{\frac{1}{2}}}{\phi_{T} T_{H}-\left(\kappa_{1} \phi_{T} T_{H} T_{L} / \kappa_{2}\right)^{\frac{1}{2}}} .
\end{aligned}
$$

Eq. (34) is the finite-time exergoeconomic performance bound of the irreversible combined refrigeration cycle with losses of heat resistance and internal irreversibility.

If $\phi_{T}=1$ and $q=0$, Eqs. (23), (25), (24) and (26) become

$$
\begin{gathered}
\left\{\left[\varepsilon\left(\kappa_{2}-\kappa_{1}\right)-\kappa_{1}\right] T_{L}-\pi \varepsilon B_{1}^{*}\right\}(\pi \varepsilon+\pi)-T_{H} \pi \varepsilon\left[\varepsilon\left(\kappa_{2}-\kappa_{1}\right)-\kappa_{1}\right]=0 \\
R_{\pi}=\frac{T_{L}-\left(\kappa_{1} T_{H} T_{L} / \kappa_{2}\right)^{\frac{1}{2}}}{B_{1}^{*}} \\
\pi_{\max }=\frac{\left[T_{L}-\left(\kappa_{1} T_{H} T_{L} / \kappa_{2}\right)^{\frac{1}{2}}\right]\left[\left(\kappa_{1} \kappa_{2} T_{H} T_{L}\right)^{\frac{1}{2}}-\kappa_{1} T_{H}\right]}{B_{1}^{*}\left(\kappa_{1} T_{H} T_{L} / \kappa_{2}\right)^{\frac{1}{2}}} \\
\varepsilon_{\pi}=\frac{\left(\kappa_{1} T_{H} T_{L} / \kappa_{2}\right)^{\frac{1}{2}}}{T_{H}-\left(\kappa_{1} T_{H} T_{L} / \kappa_{2}\right)^{\frac{1}{2}}},
\end{gathered}
$$

where $B_{1}^{*}=\left[\left(1 / K_{1}\right)^{1 / 2}+\left(1 / K_{2}\right)^{1 / 2}+\left(1 / K_{3}\right)^{1 / 2}\right]^{2} / F_{T}$.

Eqs. (38) is the finite-time exergoeconomic performance bound of the endoreversible combined refrigeration cycle.

The finite-time exergoeconomic performance bound at the maximum profit rate is different from the classical reversible bound and the finite-time thermodynamic bound at the maximum cooling load. It is dependent on $T_{H}, T_{L}, T_{0}, \phi_{1}, \phi_{2}$ and $\psi_{2} / \psi_{1}$. Note that for the process to be potential profitable, the following relationship must exist: $0<\psi_{2} / \psi_{1}<1$, because one unit of power input must give rise to at least one unit of exergy output rate.

As the price of exergy output rate becomes very large compared with that of the power input, i.e. $\psi_{2} / \psi_{1} \rightarrow 0$, Eq. (22) becomes

$$
\pi=\psi_{1}\left(\eta_{1} q+\eta_{2} R\right)-\frac{\psi_{1} \eta_{1} \phi_{T} T_{H}}{T_{L} /(R+q)-B^{*}} .
$$


When $T_{H} \rightarrow T_{o}$, Eq. (39) becomes $\pi=\psi_{1} \eta_{2} R$. It indicates that the profit rate maximization approaches the cooling load maximization (Chen et al 1999b).

On the other hand, as the price of exergy output rate approaches the price of the power input, i.e. $\psi_{2} / \psi_{1} \rightarrow 1$, equation (22) becomes

$$
\pi=-\psi_{1} T_{o}\left\{\frac{\phi_{T} T_{H}(R+q)-q\left[T_{L}-(R+q) B^{*}\right]}{T_{H}\left[T_{L}-(R+q) B^{*}\right]}-\frac{R}{T_{L}}\right\}=-\psi_{1} T_{o} \sigma,
$$

where $\sigma$ is entropy production rate of the combined refrigeration cycle. That is the profit rate maximization approaches the entropy production rate minimization, in other word, the minimum exergy loss. Equation (40) indicates that the combined refrigeration cycle is not profitable regardless of the COP at which the combined refrigeration cycle is operating. Only the combined refrigeration cycle is operating reversibly $\left(\varepsilon_{\pi}=\varepsilon_{C}=T_{L} /\left(T_{H}-T_{L}\right)\right)$ will the revenue equal to the cost, and then the maximum profit rate will be equal to zero. The corresponding entropy production rate is also zero.

Therefore, for any intermediate values of $\psi_{2} / \psi_{1}$, the finite-time exergoeconomic performance bound $\left(\varepsilon_{\pi}\right)$ lies between the finite-time thermodynamic performance bound and the reversible performance bound. $\varepsilon_{\pi}$ is related to the latter two through the price ratio $\psi_{2} / \psi_{1}$.

For a combined refrigeration cycle, which is formed by several irreversible Carnot refrigeration cycles, with losses of heat resistance, bypass heat leakage and internal irreversibilities, the optimal performance characteristics have the same expressions as equations (21)-(26), simply replace $B^{*}$ by $B_{2}^{*}$.

$$
B_{2}^{*}=\left\{\frac{1}{F_{T}} \sum_{i=1}^{n+1}\left(\prod_{j=i}^{n+1} \phi_{j} / K_{i}\right)^{1 / 2}\right\}^{2}
$$

where $n$ is the number of stages of the combined refrigeration cycle, $K_{i}$ is the heat transfer coefficient for the $i$ th stage heat exchanger between working fluids or between working fluid and the reservoirs, and $\phi_{j}$ is the internal dissipation for the $j$ th stage refrigeration cycle and $\phi_{n+1}=1$ is taken.

For the combined cycle, the optimization problem can be extended by distributing the total heat exchanger inventory $U_{T}$ (Bejan 1988, 1989; Chen et al 2004b), which is assumed to be a constant $U_{T}=U_{1}+U_{2}+U_{3}$.

Using the above constraint, the optimal distribution of the heat exchanger inventory is obtained in the following expressions:

$$
\begin{aligned}
& \left(U_{1} / U_{T}\right)_{\mathrm{opt}}=\phi_{T}^{1 / 2} /\left(1+\phi_{2}^{1 / 2}+\phi_{T}^{1 / 2}\right) \\
& \left(U_{2} / U_{T}\right)_{\mathrm{opt}}=\phi_{2}^{1 / 2} /\left(1+\phi_{2}^{1 / 2}+\phi_{T}^{1 / 2}\right) \\
& \left(U_{3} / U_{T}\right)_{\mathrm{opt}}=1 /\left(1+\phi_{2}^{1 / 2}+\phi_{T}^{1 / 2}\right) .
\end{aligned}
$$

The optimal performance characteristics have the same expressions as equations (21)-(26), again, simply replace $B^{*}$ by $B_{3}^{*}$.

$$
B_{3}^{*}=\left(1+\phi_{2}^{1 / 2}+\phi_{T}^{1 / 2}\right) / U_{T} .
$$


For a combined cycle formed by $n$ stages of irreversible refrigeration cycles, $B^{*}$ should be replaced by $B_{4}^{*}$.

$$
B_{4}^{*}=\frac{1}{U_{T}}\left\{\sum_{i=1}^{n+1}\left[\prod_{j=i}^{n+1} \phi_{j}\right]^{1 / 2}\right\}^{2}
$$

\section{Numerical examples}

To illustrate the preceding analysis, numerical examples are provided. In the calculations, it is set that $T_{H}=300 \mathrm{~K}, T_{L}=260 \mathrm{~K}, T_{0}=290 \mathrm{~K}, K_{1}=K_{2}=K_{3}, K_{1} F_{T}=10 \mathrm{~kW} / K$, $\psi_{1}=1000$ yuan $/ \mathrm{kW}, \phi_{1}=\phi_{2}=1$ or $1 \cdot 1$, and $q=C_{i}\left(T_{H}-T_{L}\right.$ ) (same as the work of Bejan $(1988,1989))$ where $C_{i}=0$ or $0.04 \mathrm{~kW} / \mathrm{K}, C_{i}$ is the thermal conductance inside the combined refrigeration cycle.

Figure 2 shows the effects of $\psi_{2} / \psi_{1}$ on the optimal profit rate $\pi$ versus the cooling load $R$ characteristic with $C_{i}=0.04 \mathrm{~kW} / \mathrm{K}$ and $\phi_{1}=\phi_{2}=1.1$. It illustrates that the profit rate $\pi$ is a monotonic increasing function of cooling load $R$ with $0 \leq \psi_{2} / \psi_{1} \leq 0.033$, which indicates that the refrigerator is profitable regardless of the cooling load is at which the refrigerator is operating. When $0.033<\psi_{2} / \psi_{1}<0.393$, the curves of $\pi-R$ are paraboliclike ones, which means there exists the maximum profit rate point. The optimal profit rate $\pi$ is a monotonic decreasing function of cooling load $R$ when $\psi_{2} / \psi_{1} \geq 0.393$, which indicates that the refrigerator is not profitable regardless of the cooling load is at which the refrigerator is operating.

Figure 3 shows the relation between the optimal profit rate $\pi$ and COP $\varepsilon$ of the combined refrigeration cycle with heat resistances and heat leakage losses. It can be seen that the curves of $\pi-\varepsilon$ change quantitatively and qualitatively due to the change of $\psi_{2} / \psi_{1}$. The qualitative differences are as following: when $0 \leq \psi_{2} / \psi_{1} \leq 0.033$, the curves of $\pi-\varepsilon$ are hyperbola-like ones, and the refrigerator is profitable regardless of the cooling load is at

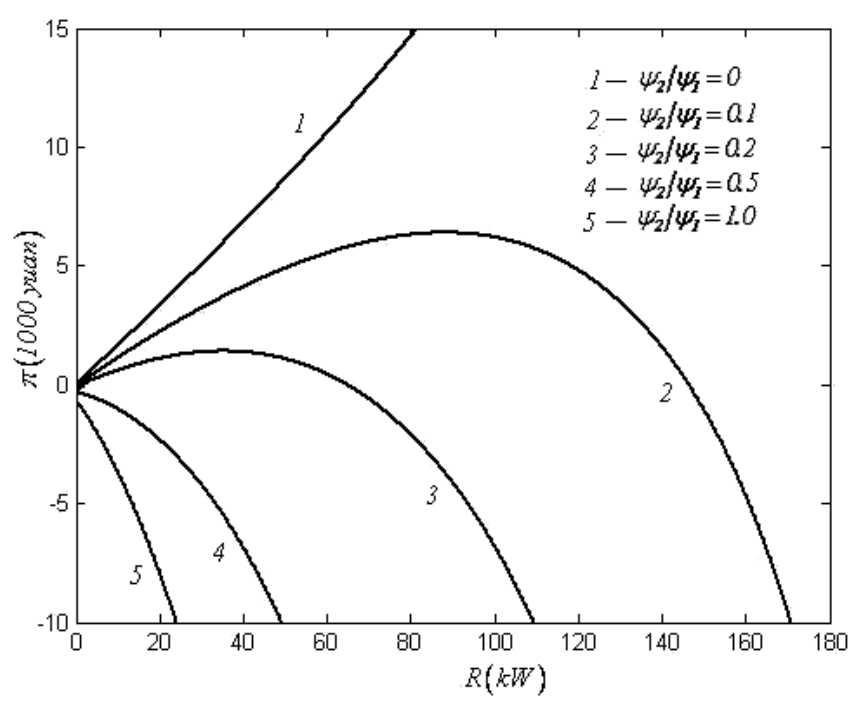

Figure 2. Effect of price ratio $\psi_{2} / \psi_{1}$ on $\pi-R$ characteristic. 


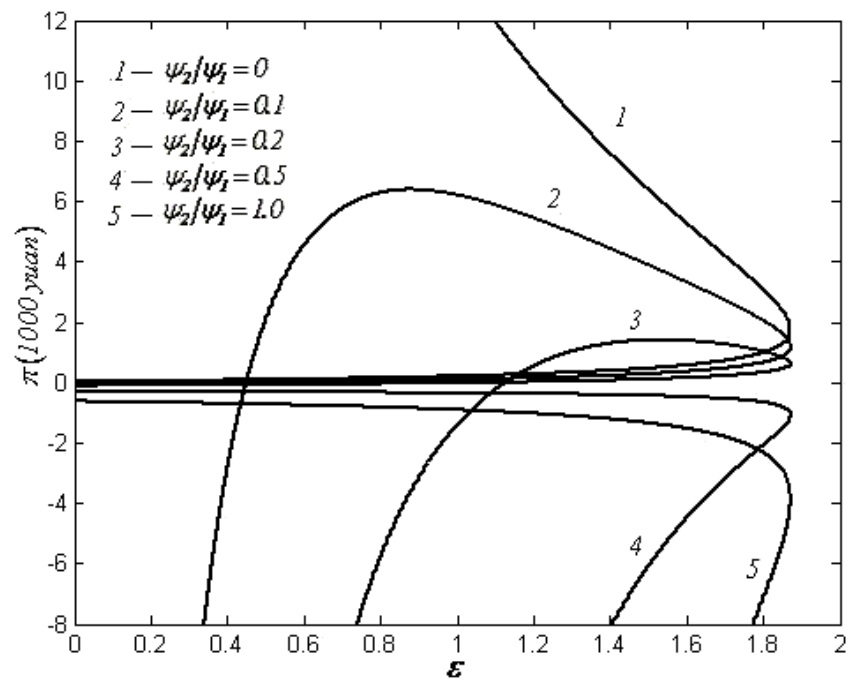

Figure 3. Effect of price ratio $\psi_{2} / \psi_{1}$ on $\pi-\varepsilon$ characteristic.

which the refrigerator is operating. When $0.033<\psi_{2} / . \psi_{1} \leq 0.393$, the curves of $\pi-\varepsilon$ are loop-shaped ones, which means that there exist a maximum optimal profit rate point and a maximum COP point. In addition, the areas enclosed by curves of $\pi-\varepsilon$ decreases with the increase of $\psi_{2} / \psi_{1}$. When $\psi_{2} / \psi_{1}>0.393$, the curves of $\pi-\varepsilon$ are hyperbola-like ones, and the refrigerator is not profitable regardless of the cooling load is at which the refrigerator is operating.

Figure 4 shows the relation between the optimal profit rate $\pi$ and COP $\varepsilon$ of the combined refrigeration cycle with different loss items and $\psi_{2} / \psi_{1}=0 \cdot 1$. It illustrates that the curves of $\pi-\varepsilon$ are parabolic-like ones in the case of $q=0$, while the curves are loop-shaped ones in the case of $q \neq 0$. It shows that the internal irreversibilities only have quantitative

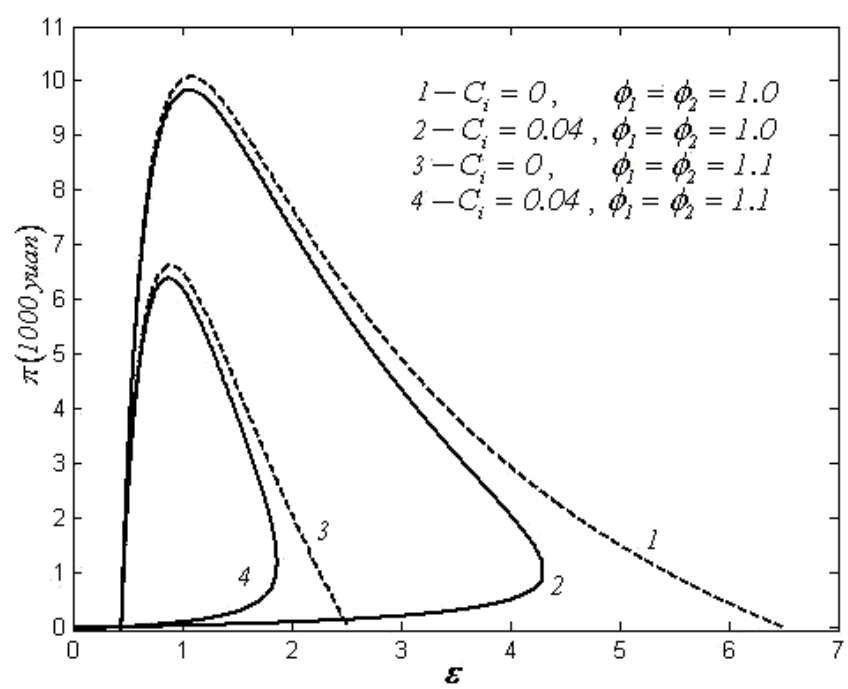

Figure 4. Effects of internal irreversibility and heat leakage on $\pi-\varepsilon$ characteristic. 


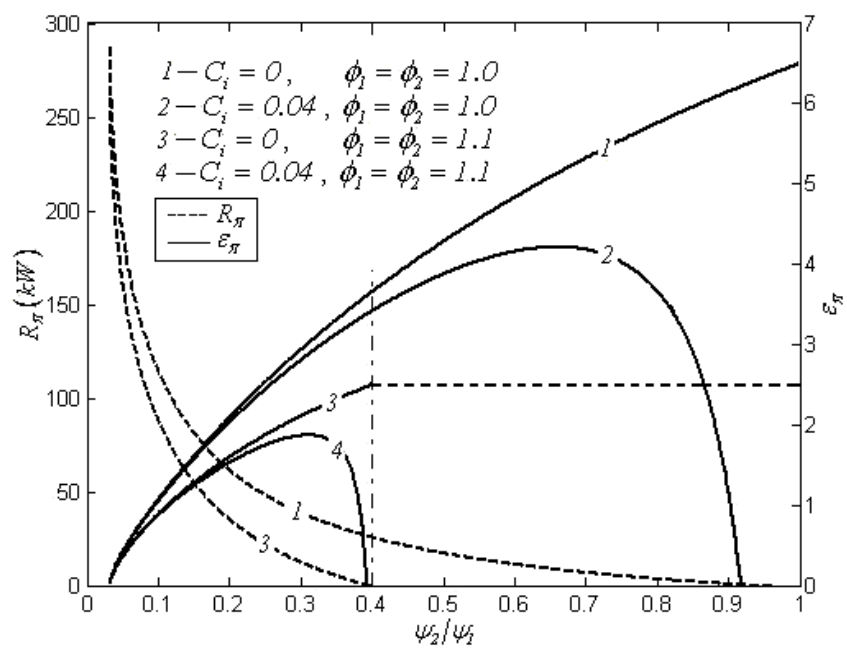

Figure 5. $\quad \varepsilon_{\pi}$ and $R_{\pi}$ versus $\psi_{2} / \psi_{1}$ characteristic.

effects on the curves of $\pi-\varepsilon$, while the heat leakage has not only quantitative effects but also qualitative effects on the curves of $\pi-\varepsilon$. In addition, because of the existence of heat leakage and internal irreversibilities, both the maximum profit rate $\left(\pi_{\max }\right)$ and the finite-time exergoeconomic performance bound $\left(\varepsilon_{\pi}\right)$ decrease.

Figures 5 and 6 show the relations between the finite-time exergoeconomic performance bound $\left(\varepsilon_{\pi}\right)$ and $\psi_{2} / \psi_{1}$, and between the maximum profit rate $\left(\pi_{\max }\right)$ and $\psi_{2} / \psi_{1}$ with different loss items. Figure 5 shows that $\varepsilon_{\pi}$ is a monotonic increasing function of $\psi_{2} / \psi_{1}$ without heat leakage, while the curves of $\varepsilon_{\pi}-\psi_{2} / \psi_{1}$ are parabolic-like ones with heat leakage. Figure 6 shows that $\pi_{\max }$ is a monotonic decreasing function of $\psi_{2} / \psi_{1}$. For the endoreversible combined refrigeration cycle, $\pi_{\max }$ is zero only when $\psi_{2} / \psi_{1}=1$. However, $\pi_{\max }$ is zero when $\psi_{2} / \psi_{1}<1$ with internal irreversibilities.

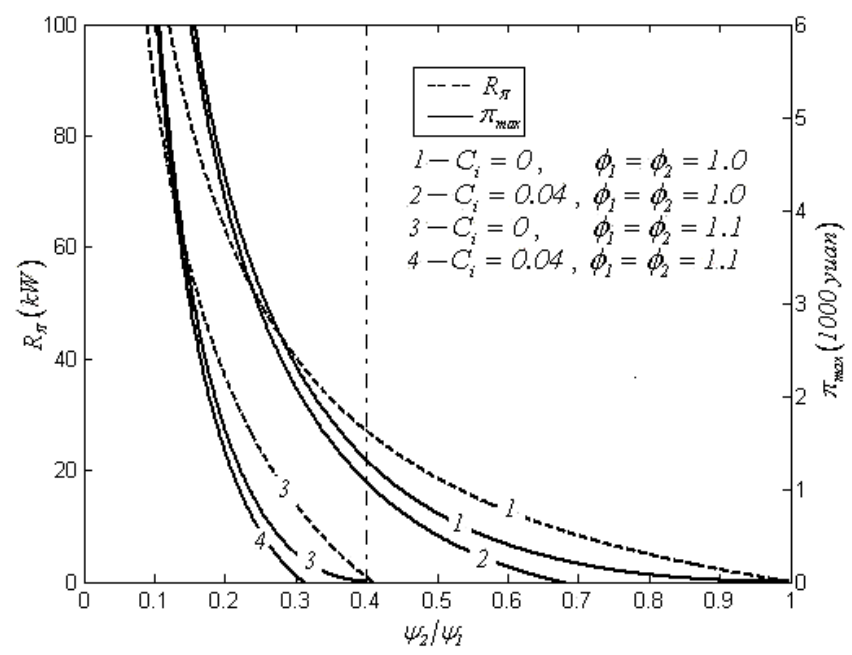

Figure 6. $\pi_{\max }$ and $R_{\pi}$ versus $\psi_{2} / \psi_{1}$ characteristic. 


\section{Conclusion}

Economics plays a major role in the refrigeration industry. This paper analyses the exergoeconomic performance of the generalized irreversible combined refrigeration cycle model. One seeks the economic optimization objective function instead of pure thermodynamic parameters by viewing the refrigerator as a production process. It is shown that the economic and thermodynamic optimization converged in the limits $\psi_{2} / \psi_{1} \rightarrow 0$ and $\psi_{2} / \psi_{1} \rightarrow 1$. When the profit rate for exergy conversion is small, the maximum profit operation is near the minimum exergy loss operation, while when the work input is very cheap compared to the price of exergy output, the maximum profit operation is near the maximum cooling load operation. The heat leakage and the internal irreversibility affect the finite time exergoeconomic performance of the combined refrigeration cycle obviously. It is necessary to investigate the optimal performance of a generalized irreversible combined refrigeration cycle.

This paper is supported by The National Natural Science Foundation of P. R. China (Project No. 10905093), Program for New Century Excellent Talents in University of P. R. China (Project No. NCET-04-1006) and The Foundation for the Author of National Excellent Doctoral Dissertation of P. R. China (Project No. 200136). The authors wish to thank the reviewers for their careful, unbiased and constructive suggestions, which led to this revised manuscript.

\section{References}

Andresen B 1983 Finite-Time Thermodynamics, Physics Laboratory II, University of Copenhagen Bejan A 1988 Theory of heat transfer-irreversible power plant. Int. J. Heat Mass Transfer 31(6): 1211-1219

Bejan A 1989 Theory of heat transfer-irreversible refrigeration plants. Int. J. Heat Mass Transfer 32(9): 1631-1639

Bejan A 1993 Power and refrigeration plants for minimum heat exchanger inventory. ASME Trans. J. Energ. Res. Tech. 115(2): 148-150

Bejan A 1996 Entropy generation minimization. The new thermodynamics of finite-size device and finite-time processes. J. Appl. Phys. 79(3): 1191-1218

Berry R S, Salamon P, Heal G 1978 On a relation between economic and thermodynamic optima. Resources and Energy 1(2): 125-137

Berry R S, Kazakov V A, Sieniutycz S, Szwast Z, Tsirlin A M 1999 Thermodynamic Optimization of Finite Time Processes. Chichester: Wiley

Chen J, Yan Z 1988 Optimal performance of an endoreversible-combined refrigeration cycle. J. Appl. Phys. 63(10): 4795-4799

Chen L, Sun F, Chen W 1991 Finite time exergoeconomic performance bound and optimization criteria for two-heat-reservoir refrigerators. Chinese Sci. Bull. 36(2): 156-157 (in Chinese)

Chen L, Sun F, Chen W 1995 Optimization of the specific rate of refrigeration in combined refrigeration cycles. Energy The Int. J. 20(10): 1049-1053

Chen L, Sun F, Wu C 1996 Maximum profit performance of an absorption refrigerator. Int. J. Energ. Environ. and Econom. 4(1): 1-7

Chen L, Wu C, Sun F 1997 Steady flow combined refrigeration cycle performance with heat leak. Appl. Thermal Eng. 17(7): 639-645

Chen L, Wu C, Sun F 1999a Finite time thermodynamic optimization or entropy generation minimization of energy systems. J. Non-Equilib. Thermodyn. 24(4): 327-359 
Chen L, Bi Y, Sun F, Wu C 1999b A generalized model of combined refrigeration plant and its performance. Int. J. Therm. Sci. 38(8): 712-718

Chen L, Wu C, Sun F 2001 Effect of heat transfer law on finite time exergoeconomic performance of a Carnot refrigerator. Exergy An Int. J. 1(4): 295-302

Chen L, Sun F 2004 Advances in finite time thermodynamics analysis and optimization. New York: Nova. Science Publishers

Chen L, Sun F, Wu C 2004a Maximum profit performance for generalized irreversible Carnot engines. Appl. Energy 79(1): 15-25

Chen L, Sun F, Wu C 2004b Optimum allocation of heat exchanger area for refrigeration and air conditioning plants. Appl. Energy 77(3): 339-354

Chen L 2005 Finite-Time Thermodynamic Analysis of Irreversible Processes and Cycles. Higher Education Press, Beijing

Chen L, Sun F, Wu C 2005 Endoreversible thermoeconomics for heat engines. Appl. Energy 81(4): 388-396

De Vos A 1995 Endoreversible thermoeconomics. Energ. Convers. and Manage. 36(1): 1-5

De Vos A 1997 Endoreversible economics. Energ. Convers. and Manage. 38(4): 311-317

Durmayaz A, Sogut O S, Sahin B, Yavuz H 2004 Optimization of thermal systems based on finitetime thermodynamics and thermoeconomics. Progress on Energy \& Combustion Science 30(2): $175-217$

El-Sayed M 2003 The Thermoeconomics of Energy Conversion. London: Elsevier

Goktun S 1996 Coefficient of performance for an irreversible combined refrigeration cycle. Energy The Int. J. 21(7/8): 721-724

Ibrahim O M, Klein S A, Mitchell J W 1992 Effects of irreversibility and economics on the performance of a heat engine. ASME Trans. J. Sol. Energy Engng. 114(4): 267-271

Kodal A, Sahin B, Yilmaz T 2000a Effects of internal irreversibility and heat leakage on the finite time thermoeconomic performance of refrigerators and heat pumps. Energ. Convers. and Manage. 41(6): 607-619

Kodal A, Sahin B, Oktem A S 2000b Performance analysis of two stage combined heat pump system based on thermoeconomic optimization criterion. Energ. Convers. and Manage. 41(18): 1989-2008

Kodal A, Sahin B, Erdil A 2002 Performance analysis of a two-stage irreversible heat pump under maximum heating load per unit total cost conditions. Int. J. Exergy 2(3): 159-166

Kodal A, Sahin B, Ekmekci I, Yilmaz T 2003 Thermoeconomic optimization for irreversible absorption refrigerators and heat pumps. Energ. Convers. and Manage. 44(1): 109-123

Kodal A, Sahin B 2003 Finite time thermoeconomic optimization for irreversible heat engines. Int. J. Therm. Sci. 42(8): 777-782

Qin X, Chen L, Sun F, Wu C 2005 Thermoeconomic optimization of an endoreversible four-heatreservoir absorption-refrigerator. Appl. Energy 81(4): 420-433

Sahin B, Kodal A 1999 Finite time thermoeconomic optimization for endoreversible refrigerators and heat pumps. Energ. Convers. and Manage. 40(9): 951-960

Sahin B, Kodal A, Koyun A 2001 Optimal performance characteristics of a two-stage irreversible combined refrigeration system under maximum cooling load per unit total cost conditions. Energ. Convers. and Manage. 42(4): 451-465

Sahin B, Kodal A 2001 Performance analysis of an endoreversible heat engine based on a new thermoeconomic optimization criterion. Energ. Convers. and Manage. 42(9): 1085-1093

Sahin B, Kodal A 2002 Thermoeconomic optimization a two-stage combined refrigeration system: A finite time approach. Int. J. Refrig. 25(7): 872-877

Salamon P, Nitzan A 1981 Finite time optimizations of a Newton's law Carnot cycle. J. Chem. Phys. 74(6): 3546-3560

Sieniutycz S, Salamon P 1990 Advances in Thermodynamics, Volume 4: Finite Time Thermodynamics and Thermoeconomics. New York: Taylor \& Francis

Tsatsaronts G 1993 Thermoeconomic analysis and optimization of energy systems. Progress on Energy and Combustion Science 19(3): 227-257 
Wu C, Chen L, Sun F 1996 Effect of heat transfer law on finite time exergoeconomic performance of heat engines. Energy The Int. J. 21(12): 1127-1134

Wu C, Chen L, Sun F 1998 Effect of heat transfer law on finite time exergoeconomic performance of a Carnot heat pump. Energ. Convers. and Manage. 39(7): 579-588

Wu F, Chen L, Sun F, Wu C 2000 Finite-time exergoeconomic performance bound for a quantum Stirling engine. Int. J. Eng. Sci. 38(2): 239-247

Zheng Z, Chen L, Sun F, Wu C 2006 Maximum profit performance for a class of universal steady flow endoreversible heat engine cycles. Int. J. Ambient Energy 27(1): 29-36 